\title{
Pankreas Kanseri Radyoterapisinde 3 Farklı Tedavi Tekniğinin Dozimetrik Karşılaştırılması: Retrospektif Çalışma
}

\author{
Burcu İBİCIOĞLU, Meral KURT, Sema GÖZCÜ TUNÇ, \\ Candan DEMİRÖZ ABAKAY, Sibel KAHRAMAN ÇETINTAŞ, Abdulhamit TURAN, \\ Ece Ayfer CURA, Habibe ALTAŞ
}

Uludağ Üniversitesi Tıp Fakültesi, Radyasyon Onkolojisi Anabilim Dalı, Bursa.

\begin{abstract}
ÖZET
Bu çalışmada 2012-2017 yılları arasında kliniğimizde definitif radyoterapi uygulanmış, primer pankreas başı tümörü olan inopere hastalar retrospektif olarak değerlendirilmiștir. Her hastaya tümör, tümör çevresi ve bölgesel lenf nodlarına yönelik 3 farklı tedavi tekniği kullanılarak 5 farklı planlama yapılmış, tüm planlar hedef hacim dozları 54/45 Gy olacak şekilde seçilmişsir. Planlar Volümetrik Ark Tedavi (VMAT) ve Yoğunluk Ayarlı Radyoterapi (IMRT) için 6 MV X-ışıı, 3 Boyutlu Konformal Radyoterapi (3BKRT) için 6/15 MV X-1şını kullanılarak hazırlanmıştır. Planlanan hedef hacim dozları (PTV), Konformite indeksi (CI), Homojenite indeksi (HI), riskli organlar (böbrekler, spinal kord, karaciğer) açısından teknikler karşılaştırılmıştı. PTV $_{54}$ ve PTV $_{45}$ için ortalama CI indeksleri VMAT 1 ark planlarında sırasıyla 1,16 ve 1,03, VMAT 2 arkda ise 1,01 ve 1,00 bulunmuştur. CI açısından hedef hacimler arasında anlamlı fark olmamasına rağmen en uygun CI değeri VMAT 2 ark planlarında bulunmuştur $\left(\mathrm{PTV}_{45}\right.$ için $\mathrm{p}=0,806, \mathrm{PTV}_{54}$ için $\left.\mathrm{p}=0,595\right)$. IMRT ile 3BKRT teknikleri arasında CI değeri açısından fark bulunamamış, en uygun değer IMRT tekniğinde elde edilmiştir. Kritik organ dozları açısından VMAT 1 ark ve VMAT 2 ark planları arasında istatistiksel farklılık olmamasına rağmen VMAT 2 ark planlarının dozları daha düşük bulunmuştur. Pankreas tümörleri yerleşim yeri olarak böbrekler ve karaciğer gibi kritik organlara yakın oluşundan 3BKRT planları karaciğer açısından kabul edilebilir; ancak böbrekleri koruma açısından yeterli değildir. VMAT 2 ark tekniği diğer tekniklere göre dozun hedefi sarımı, CI, HI ve kritik organ dozlarında üstünlük sağlamıştır. Bu yüzden yan etkiler göz önüne alındığında tedavi tekniği olarak VMAT tercih edilebilir.

Anahtar Kelimeler: VMAT. Pankreas Kanseri. 3BKRT. IMRT. Radyoterapi.
\end{abstract}

Dosimetric Comparison of 3 Different Treatment Techniques in Pancreatic Cancer Radiotherapy: Retrospective Study

\begin{abstract}
In this study, inoperable patients with primary pancreatic head tumor who underwent definitive radiotherapy in our clinic between 2012-2017 were evaluated retrospectively. Five different plannings were done using 3 different treatment techniques for tumor, tumor periphery and regional lymph nodes in each patient, and plans were selected so that the target volume doses would be 54/45 Gy. Volumetric Arc Therapy (VMAT) and Intensity Modulated Radiotherapy (IMRT) plans were prepared using 6 MV X-rays and for 3DCRT plans 6/15 MV X-rays were used. Techniques were compared in terms of planned target volume doses (PTV), conformity index (CI), homogeneity index (HI), organs at risk (kidneys, spinal cord, liver). The CI indices for PTV $\mathrm{PT}_{54}$ and $\mathrm{PTV}_{45}$ were found to be 1.16 and 1.03 for VMAT 1 arc plans and 1.01 and 1.00 for VMAT 2 arc, respectively. The optimal CI value was found in the VMAT 2 arc plans, although there was no significant difference between the target volumes in terms of CI $\left(\mathrm{PTV}_{45} \mathrm{p}=0,806-\mathrm{PTV}_{54} \quad \mathrm{p}=0,595\right)$. There was no difference in terms of CI value between IMRT and 3D-CRT techniques, and the most appropriate value was obtained by IMRT technique. Doses of VMAT 2 arc plans were found to be lower, although there was no statistical difference between VMAT 1 arc and VMAT 2 arc plans in terms of critical organ doses. Since pancreatic tumors are close to critical organs such as the kidneys and liver, 3DCRT plans can be accepted in terms of liver; but not enough for the kidney protection. VMAT 2 arc technique was superior in dose targeting, CI, HI and critical organ doses when all tecniques are compared. Therefore, VMAT may be a preferred treatment technique when side effects are taken into account.
\end{abstract}

Key Words: VMAT. Pancreatic Cancer. 3D-CRT. IMRT. Radiotherapy.

Geliş Tarihi: 6 Haziran 2018

Kabul Tarihi: 16 Temmuz 2018

Yüksek Lisans Öğrencisi, Burcu İBicioĞLU

Uludağ Üniversitesi Hastanesi

Radyasyon Onkolojisi, Anabilim Dalı

NILÜFER/BURSA

Tel.: 05389720540

E-posta: burcuibicioglu@gmail.com
Görülme sıklığı bakımından ülkemizde 12. sırada yer alan pankreas kanseri tüm kanserlerin \%2'sini oluşturmaktadır ${ }^{1}$. Yüksek ölüm oranlarına ve agresif bir yapıya sahip olan bu kanserin 5 yıllık sağkalım oranı yaklaşık \%6' dir ${ }^{2,3}$. Prognozu belirlemek için AJCC (American Joint Commitee on Cancer) evreleme sistemi kullanılsa da kanserin nasıl seyredeceği ve sağka- 
lımın nasıl olacağı öngörülememektedir ${ }^{4}$. Tedavi yaygin olarak cerrahi, kemoterapi ve radyoterapi (RT) gibi kombine tedavi yaklaşımlarını içerir ${ }^{5}$. Lokal ileri pankreas kanserli hastalar için, kemoterapinin yanında radyoterapinin sağkalımı iyileştirdiği, bununla birlikte bazı çalışmalarda sadece kemoterapi verilmesi durumunda sağkalıma etkisi olmaksızın toksisitenin arttığ gösterilmiştir ${ }^{6,7}$.

Pankreas kanserinde tümör radyasyona dirençli olmamasına rağmen, genellikle mikrometastaz riski ve bölgesel lenf düğümlerini de kapsayan geniş tedavi alanları, RT dozunu sınırlandıran çevre organlara (böbrekler, karaciğer, bağırsak, spinal kord) yakınlığı gibi sebeplerden dolayı RT için zorlu bir hedeftir ${ }^{8-10}$. Genel olarak üç boyutlu konformal radyoterapide (3BKRT) 4 alan tekniği yaygın olarak kullanılır, ancak gerekli radyasyon alanı böbrekler, mide, ince bağırsak ve karaciğer gibi kritik organların önemli miktarını da kapsar ${ }^{11}$. Tümörün konumuna bağlı kritik organlara yakın olmasından dolayı toksisite yaygın görülen bir durumdur. Randomize çalışmalarda, hastaların 3BKRT ile tedavisinde Radiation Therapy Oncology Group (RTOG) derece 3 ile 4 toksisite (mide bulantıs1, kusma ve ishal oranının sirasıyla \%11 ve \%17) görülme sıklığının yüksek olduğu saptanmış$\operatorname{tır}^{12}$. Yeni RT tekniklerinin kullanımı, toksisiteyi arttırmadan yüksek RT dozlarının güvenle uygulanmas1na izin vermektedir. Yoğunluk Ayarlı Radyoterapi (IMRT), tedavi alanı içinde dinamik doz değişiklikleri yapabilen ve hedef dokular dişındaki dokulara fazla doz vermeden tümör dozunu arttırabilmeye olanak veren bir yöntemdir. Yapılan çalışmalarda IMRT tekniği ile, 3BKRT'ye oranla hem tümör hedef hacminde daha iyi doz dağılımı, hem de kritik normal dokularda daha düşük doz sağlanabildiği gösterilmiştir. IMRT'deki bu dozimetrik avantaj tümör kontrolü üzerine olumsuz etkisi olmaksızın normal dokuları anlamlı şekilde koruyarak geç yan etkiyi azaltmakta$\mathrm{d}^{13}{ }^{13}$. Yoğunluk ayarlı radyoterapinin farklı bir versiyonu olan Volümetrik Ark Terapi (VMAT) ise son dönemde akciğer, prostat, baş-boyun ve anüs kanserlerinin tedavisinde kullanılarak klinik uygulamalarda yerini almıştır. Yapılan çalışmalarda VMAT tekniğinin farklı kanser türlerine yönelik RT planlaması konusunda IMRT'ye kıyasla plan kalitesini düşürmeden tedavi süresini kısaltabileceği gösterilmiştir ${ }^{14}$. VMAT, farklı yoğunluklardan oluşan bir çok 1şın demeti kullanması ve her 1şın demetinin kendi içerisinde yoğunluğunu değiştirmesiyle hedef hacim üzerinde daha homojen doz dağılımı sağlamaktadı1 ${ }^{15}$.

Bu çalışmada kliniğimizde pankreas kanseri tanısı ile radyoterapi uyguladığımız olguların farklı teknikler kullanılarak hedef hacim ve kritik organ dozlarının dozimetrik olarak karşılaştırılması değerlendirilmiştir.

\section{Gereç ve Yöntem}

\section{Hasta Seçimi, Konturlama, Doz Sınırlamaları}

Bu çalışmada Uludağ Üniversitesi Tıp Fakültesi Radyasyon Onkolojisi Anabilim Dalında tedavi görmüş, primer tümör bölgesi pankreas başı olan inopere 20 hasta Uludağ Üniversitesi Etik Kurul'u tarafindan 10 Nisan 2018 tarihli 2018-7/15 nolu karar ile seçilerek retrospektif olarak incelenmiştir. Bu hastaların; planlama tomografi kesitleri üzerine radyasyon onkoloğu tarafından RTOG protokollerine uygun Görülebilir Tümör Hacmi (GTV), Klinik Hedef Hacim (CTV) ve kritik organların (spinal kord, sağ ve sol böbrek, karaciğer) konturlamaları yapılmıştır. $\mathrm{CTV}_{45}$ bölgesel lenf nodlarını; pankreatik, duodenal, suprapankreatik, çölyak ve hepatik porta lenf nodlarını içerirken, $\mathrm{GTV}_{54}$ ise radyolojik yöntemlerle görülebilir tümör hacmini kapsamaktadır. "Set-up" hatalarını ve organ hareketlerini ortadan kaldırmak için çizilen CTV'ye 0,5 cm marj verilerek planlanan hedef hacimler (PTV) oluşturulmuştur. Tüm hastalar için günlük fraksiyon dozu 1,8 Gy ve toplam doz 54 Gy olacak şekilde tedavi planlamaları yapılmıştır. PTV'lerin çizimi sırasında hedef tümör volümünün etrafı sarılırken, kritik organlardan (spinal kord, karaciğer, sağ ve sol böbrek) kaçınılmaya dikkat edilmiştir.

Öncelik planlanan hedef hacmin \%98'inin verilmek istenen dozun \%98'ini alması ve planın maksimum dozunun verilen dozun \%110'unu geçmemesini sağlamaktır.

Radyoterapide hedef hacme istenilen doz verilirken tümöre yakın kritik organların korunması önemlidir. Planlarımızı oluştururken kritik organlar için doz sinırlamalarımız Quantitative Analyses of Normal Tissue Effects in the Clinic'e (QUANTEC) göre olup Tablo I'de verilmiştir.

Tablo I. Kritik organ doz sinırlamaları

\begin{tabular}{|ccc|}
\hline Böbrekler & Karaciğer & Spinal kord \\
$\mathrm{V}_{12 G \mathrm{y}}<55(\%)$ & $\mathrm{V}_{27 G \mathrm{G}}<35(\%)$ & D $_{\text {maks }}<45 \mathrm{~Gy}$ \\
$\mathrm{~V}_{18 G \mathrm{y}}<35(\%)$ & $\mathrm{V}_{35 \mathrm{~Gy}}<66(\%)$ & \\
D $_{\text {ort }}<18 \mathrm{~Gy}$ & D $_{\text {ort }}<28 \mathrm{~Gy}$ & \\
\hline
\end{tabular}

\section{Planların Hazırlanması}

\section{3 alan ve 4 alan $3 B K R T$}

Tedavi planları CMS (Computerized medical systems) XIO TPS ile Siemens Artiste lineer akseleratörüne göre hazırlanmıştır. Planlar öne doğru planlama (forward planing) tekniği kullanılarak yapılmış ve 6/15 Mv X-şını kullanılmıştır. Üç alan tekniği için $0^{0}$, $90^{\circ}, 270^{\circ}$ sşın açıları kullanılarak planlar hazırlanmıştır. Dört alan tekniğinde ise 1 şın açıları $0^{\circ}, 90^{\circ}, 180^{\circ}, 270^{\circ}$ olarak kullanılmıştır. Bazı durumlarda istenilen doz 


\section{Pankreas Kanserinde Radyoterapi Tedavisi}

dağılımını sağlamak için "wedge” kullanılmıştır. Planların tümü "superposition" algoritması kullanılarak hazırlanmıştır. Planlamalar yapılırken ilk olarak 25 fraksiyonda hedef volüm $\mathrm{CTV}_{45}$ 'e $1,5 \mathrm{~cm}$ “multi-leaf kolimatör" (MLC) marjı verilerek yapılmıştır. İlk boost 3 fraksiyonda ve 540 cGy olarak hedef volüm $\mathrm{CTV}_{50}$ 'ye $0,8 \mathrm{~cm}$ MLC marjla, ikinci boost ise 2 fraksiyonda ve 360 cGy doz olarak hedef volüm GTV $_{54}$ 'de $0,5 \mathrm{~cm}$ MLC marjla yapılmıştır.

\section{VMAT ve IMRT Teknikleri}

IMRT planları CMS (Computerized medical systems) XIO TPS ile Siemens Artiste lineer akseleratörüne göre hazırlanmıştır. IMRT planları tersten planlama (inverse planing) yöntemiyle $6 \mathrm{MV}$ X-1şını kullanılarak 5 alan olacak şekilde planlanmıştır. Planların tümü "superposition" algoritması kullanılarak hazırlanmıştır. Planlar 1şın açıları $0^{\circ}, 60^{\circ}, 72^{\circ}, 90^{\circ}, 330^{\circ}$ olacak şekilde 5 alan olarak hazırlanmıştır.

VMAT planları doz optimizasyonu ve hesaplamalar için Monte Carlo algoritması kullanan Monaco TPS ile Elekta Synergy lineer akseleratörüne göre hazırlanmıştır. Oluşturulan planlarda ark başına maksimum kontrol noktası 140 ve minimum segment genişliği 1 $\mathrm{cm}$ olarak seçilmişir. Hem 1 ark hem de 2 ark planları gantri başlangıç açısı $180^{\circ}$, dönme açısı ise $360^{\circ}$ saat yönünde olacak şekilde yapılmıştır.

Planlar yapılmadan önce Biyolojik Eşdeğer Doz (BED) hesabı yapılmış, hem VMAT hem de IMRT planlar1 "Simultaneous Integrated Boost" (SIB) tekniği ile 2 Gy'den 27 fraksiyon olacak şekilde $6 \mathrm{MV}$ X1şını kullanılarak hazırlanmıştır.
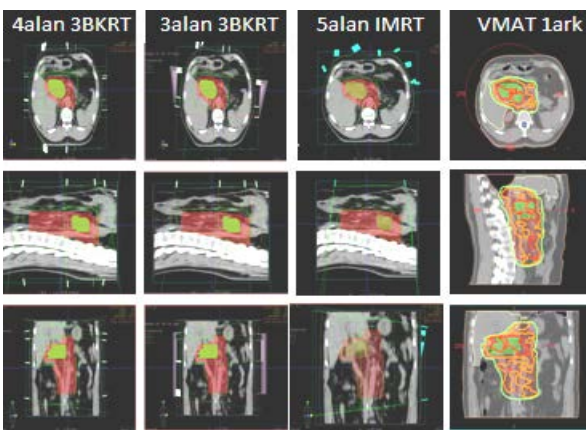

Sekil 1:

Bir hastaya ait tekniklerde aksiyel, sagital, koronal kesit görüntüleri

\section{İstatistiksel Analiz}

Statistical Package for Social Sciences (SPSS Versiyon 20) programı yardımıyla planların istatistiksel analizi yapıldı. İstatistiksel analizde normal dağılıma uygunluk gösteren değerlerde Anova, normal dağılıma uygunluk göstermeyen değerlerde Kruskal Wallis testi uyguland. Sonuç olarak $\mathrm{p}<0,005$ istatistiksel olarak anlamlı kabul edildi.

\section{Bulgular}

Tüm tedavi tekniklerinde istenilen dozun hedefi sarımı sağlanmıştır. Tablo II'de hedef hacimler için teknikler arasındaki dozimetrik parametrelerin karşılaştırılması verilmiştir. Hedef hacimlerin aldıkları ortalama doz, maksimum ve minimum dozun yanı sıra CI ile HI değerleri Tablo II'de gösterilmiştir.

Tablo III'de kritik organlar için teknikler arasındaki dozimetrik parametrelerin karşılaştırılması verilmiştir. Kritik organlar için medyan (minimum-maksimum) değerleri Tablo III'de gösterilmiştir.

Tablo II. Tümör hacimleri için VMAT, IMRT ve 3BKRT teknikleri arasındaki dozimetrik parametrelerin karşılaştırılması

\begin{tabular}{|c|c|c|c|c|c|c|}
\hline \multirow{11}{*}{ PTV $_{54}$} & & $\begin{array}{l}\text { VMAT } \\
1 \text { ark }\end{array}$ & $\begin{array}{l}\text { VMAT } \\
2 \text { ark }\end{array}$ & $\begin{array}{c}\text { 3alan } \\
\text { 3BKRT }\end{array}$ & $\begin{array}{c}\text { 4alan } \\
\text { 3BKRT }\end{array}$ & $\begin{array}{l}\text { 5alan } \\
\text { IMRT }\end{array}$ \\
\hline & $D_{\min }$ & $\begin{array}{c}54,09(50,4- \\
54,7)\end{array}$ & $\begin{array}{c}54,49(53,8 \\
55,2)\end{array}$ & $\begin{array}{l}30(52,1- \\
54,6)\end{array}$ & $\begin{array}{l}4,00 \pm(52,8 \\
-55,5)\end{array}$ & $\begin{array}{l}2,50 \pm(50,8 \\
-54,5)\end{array}$ \\
\hline & $D_{\max }$ & $\begin{array}{c}57,36(55,6- \\
59)\end{array}$ & $\begin{array}{c}57,41(55,5- \\
58,8)\end{array}$ & $\begin{array}{c}57,02(55,5- \\
59,9)\end{array}$ & $\begin{array}{c}56,94(55,2- \\
58,4)\end{array}$ & $\begin{array}{c}57,43(56,4- \\
58,5)\end{array}$ \\
\hline & $D_{\text {ort }}$ & $55,68 \pm 0,84$ & $55,82 \pm 0,59$ & $55,21 \pm 0,76$ & $55,56 \pm 0,80$ & $55,28 \pm 0,43$ \\
\hline & $\mathrm{HI}$ & $0,06 \pm 0,02$ & $0,05 \pm 0,01$ & $0,07 \pm 0,01$ & $0,05 \pm 0,01$ & $0,09 \pm 0,02$ \\
\hline & $\mathrm{Cl}$ & $\begin{array}{c}1,16(1- \\
1,93)\end{array}$ & $1,01(1-1,06)$ & $\begin{array}{c}0,99(0,8- \\
1,05)\end{array}$ & $\begin{array}{c}0,98(0,75- \\
1,05)\end{array}$ & $\begin{array}{c}0,99(0,7- \\
1,06)\end{array}$ \\
\hline & $D_{\min }$ & $\begin{array}{c}45,06(42,0- \\
45,7)\end{array}$ & $\begin{array}{c}45,76(45- \\
47,7)\end{array}$ & $\begin{array}{c}41,40(31,4- \\
47,4)\end{array}$ & $\begin{array}{c}41,80(32,6- \\
45,2)\end{array}$ & $\begin{array}{c}40,80(30- \\
45,3)\end{array}$ \\
\hline & $D_{\max }$ & $\begin{array}{c}56,96(55- \\
58,3)\end{array}$ & $\begin{array}{c}56,95(55- \\
58,3)\end{array}$ & $\begin{array}{c}56,80(55,2- \\
59,8)\end{array}$ & $\begin{array}{c}56,70(55- \\
58,1)\end{array}$ & $\begin{array}{c}56,7(55,4- \\
57,7)\end{array}$ \\
\hline & $D_{\text {ort }}$ & $\begin{array}{c}51,17(50- \\
53,6)\end{array}$ & $\begin{array}{c}51,72(50,3- \\
54,2)\end{array}$ & $\begin{array}{c}51,32(47,9- \\
54)\end{array}$ & $\begin{array}{c}51,44(47,7- \\
53,3)\end{array}$ & $\begin{array}{c}48,64(44,5- \\
51)\end{array}$ \\
\hline & $\mathrm{HI}$ & $\begin{array}{c}0,23(0,19- \\
0,28)\end{array}$ & $\begin{array}{c}0,21(0,18- \\
0,25)\end{array}$ & $\begin{array}{c}0,31(0,17- \\
0,54)\end{array}$ & $\begin{array}{c}0,30(0,20- \\
0,50)\end{array}$ & $\begin{array}{c}0,35(0,24- \\
0,57)\end{array}$ \\
\hline & $\mathrm{Cl}$ & $\begin{array}{c}1,03(1- \\
1,65)\end{array}$ & $1,00(1-1,08)$ & $\begin{array}{c}0,95(0,78- \\
1,05)\end{array}$ & $\begin{array}{c}0,96(0,80- \\
1,05)\end{array}$ & $\begin{array}{c}0,96(0,80- \\
1,05)\end{array}$ \\
\hline
\end{tabular}

Konformite Indeksi (CI) ve Homojenite Indeksi (HI) değerleri

Planların değerlendirilmesinde kullanılan Homojenite Indeksi (HI) formülü;

$\mathrm{HI}=\frac{D_{02}-D_{98}}{D_{50}}$

şeklindedir. Burada $\mathrm{D}_{2}$ hedefin \%2'sinin aldığ 1 doz, $\mathrm{D}_{98}$ hedefin \%98'inin aldığ $\mathrm{doz}, \mathrm{D}_{50}$ hedefin $\% 50$ 'sinin aldığ 1 doz miktarıdır ${ }^{16}$.

HI değerini yorumladığımızda, HI değerinin 0'a yaklaşması planlanan hedef hacmin doz dağılımının oldukça homojen olduğunu gösterir. Değer 0'dan uzaklaşırsa doz homojenitesi azalır bu nedenle yapılan plan tekrar gözden geçirilmeli ve en uygun HI değeri elde edilmelidir. Aksi halde hastaya uygulanan tedavi beklenen yararı sağlamayacaktır ${ }^{17,18}$. 


\section{B. İbicioğlu, ark.}

Tablo III. Kritik Organlar İçin Teknikler Arasındaki Dozimetrik Parametrelerin Karşılaştırılması ve $\mathrm{p}$-Değerleri

\begin{tabular}{|c|c|c|c|c|c|}
\hline $\begin{array}{c}\text { Kritik } \\
\text { Organlar }\end{array}$ & $\begin{array}{l}\text { 3-Alan } \\
\text { 3BKRT }\end{array}$ & $\begin{array}{l}\text { 4-Alan } \\
\text { 3BKRT }\end{array}$ & $\begin{array}{l}\text { 5-Alan } \\
\text { IMRT }\end{array}$ & $\begin{array}{l}\text { 1-Ark } \\
\text { VMAT }\end{array}$ & $\begin{array}{l}2 \text { 2-Ark } \\
\text { VMAT }\end{array}$ \\
\hline \multicolumn{6}{|l|}{ Karaciğer } \\
\hline \multirow[t]{3}{*}{ V27(\%) } & $\begin{array}{l}46.81(32, \\
55-61,56) \\
\end{array}$ & \begin{tabular}{|c|}
$34(21,58-$ \\
$58,46)$ \\
\end{tabular} & $\begin{array}{c}28.27(17,60 \\
-38,34) \\
\end{array}$ & \begin{tabular}{|c|}
$25(20,06-$ \\
$29,92)$ \\
\end{tabular} & $\begin{array}{c}23.73(17,55 \\
29,45)\end{array}$ \\
\hline & a: 0.004 & b: 0.001 & c: 0.001 & $\mathrm{~d}: 0.001$ & e: 0.007 \\
\hline & $f: 0.001$ & $\mathrm{~g}: 0.001$ & h: 0.074 & I: 0.013 & j:0.250 \\
\hline \multirow[t]{3}{*}{ V35(\%) } & $\begin{array}{l}21.54(10, \\
75-46,33)\end{array}$ & $\begin{array}{c}11,89(8-03- \\
23,69)\end{array}$ & $\begin{array}{c}15.40(7,12- \\
26,20)\end{array}$ & \begin{tabular}{|c|}
$13.27(6,65-$ \\
$22,86)$
\end{tabular} & $\begin{array}{c}12,4(7,07- \\
22,10)\end{array}$ \\
\hline & a: 0.006 & b: 0.015 & c: 0.001 & $\mathrm{~d}: 0.001$ & e: 0.512 \\
\hline & f: 0.683 & g: 0.486 & h: 0.161 & i: 0.074 & j: 0.345 \\
\hline \multirow[t]{3}{*}{ Ort(Gy) } & $\begin{array}{l}24,38(17, \\
07-28,28)\end{array}$ & $\begin{array}{c}20,22(17,0 \\
6-26,35)\end{array}$ & $\begin{array}{c}20,55(16,20 \\
-24,16)\end{array}$ & $\begin{array}{c}19,30(16,42 \\
-21,74)\end{array}$ & $\begin{array}{c}9,15(16,29- \\
22,06)\end{array}$ \\
\hline & a: 0,056 & b: 0,021 & c: 0,001 & $\mathrm{~d}: 0,001$ & e: 0,683 \\
\hline & $f: 0,081$ & $\mathrm{~g}: 0,089$ & h: 0,098 & I: 0,161 & j: 0,806 \\
\hline \multicolumn{6}{|l|}{ Sol Böbrek } \\
\hline \multirow[t]{3}{*}{ V12Gy(\%) } & $\begin{array}{c}30.19(5,9 \\
9-73,35)\end{array}$ & $\begin{array}{c}37.90(4,69 \\
66,46)\end{array}$ & $\begin{array}{c}56.61(11,46 \\
-76,30)\end{array}$ & $\begin{array}{c}51.06(44,69 \\
-59,76)\end{array}$ & $\begin{array}{c}0.16(40,25- \\
59,86)\end{array}$ \\
\hline & a: 0.902 & b: 0.033 & $c=0,003$ & $d=0,009$ & $\mathrm{e}=0,026$ \\
\hline & $f: 0.001$ & g: 0.003 & h: 0.512 & i: 0.412 & j: 0.567 \\
\hline \multirow[t]{3}{*}{ V18Gy(\%) } & $\begin{array}{c}15.69(2,0 \\
9-56,61) \\
\end{array}$ & $\begin{array}{c}27.48(1,57- \\
47,87)\end{array}$ & $\begin{array}{c}30.88(3,14- \\
46,49)\end{array}$ & $\begin{array}{c}23.8820,06 \\
28,79)\end{array}$ & $\begin{array}{c}2.75(18,33- \\
25,37)\end{array}$ \\
\hline & a: 0,375 & b: 0,375 & c: 0,375 & $\mathrm{~d}: 0,375$ & e: 0,375 \\
\hline & $f: 0,375$ & g: 0,375 & h: 0,375 & i: 0,375 & j: 0,375 \\
\hline \multirow[t]{3}{*}{ Ort(Gy) } & $\begin{array}{c}10,73(4,3 \\
0-22,12) \\
\end{array}$ & $\begin{array}{c}11,39(3,27- \\
20,02) \\
\end{array}$ & $\begin{array}{c}14,75(7,68- \\
17,28)\end{array}$ & $\begin{array}{c}13,74(13,02 \\
-14,50) \\
\end{array}$ & $2 \begin{array}{c}13,83(12,92 \\
14,93)\end{array}$ \\
\hline & $a=1$ & $\mathrm{~b}=0,106$ & $c=0,089$ & $d=0,089$ & $e=0,023$ \\
\hline & $f=0,029$ & $g=0,021$ & $\mathrm{~h}=0,250$ & $\mathrm{I}=0,325$ & $j=0,436$ \\
\hline \multicolumn{6}{|c|}{ Sağ Böbrek } \\
\hline \multirow[t]{3}{*}{ V12Gy(\%) } & \begin{tabular}{|c|}
$74.06(18$, \\
$31-100)$
\end{tabular} & \begin{tabular}{|c|}
$71.47(7,24-$ \\
$100)$
\end{tabular} & $\begin{array}{c}57.33(29,73 \\
-87,58)\end{array}$ & $\begin{array}{c}47.09(40,09 \\
-60,85) \\
\end{array}$ & $\begin{array}{c}94.81(41,61- \\
54,60)\end{array}$ \\
\hline & a: 0.345 & b: 0.116 & c: 0.002 & $\mathrm{~d}: 0.001$ & e: 0.389 \\
\hline & $f: 0.004$ & $\mathrm{~g}: 0.001$ & h: 0.098 & i: 0.029 & j: 0.187 \\
\hline \multirow[t]{3}{*}{ V18Gy(\%) } & $\begin{array}{c}42,11(8,1 \\
9-71,11)\end{array}$ & $\begin{array}{c}50,02(2,99 \\
100)\end{array}$ & $\begin{array}{c}33,96(11,44 \\
-60,08)\end{array}$ & $\begin{array}{c}23,36(18,37 \\
-28,42)\end{array}$ & $\begin{array}{c}725,06(18,84- \\
28,15)\end{array}$ \\
\hline & $a=0,202$ & $\mathrm{~b}=0,389$ & $c=0,003$ & $d=0,003$ & $\mathrm{e}=0,021$ \\
\hline & $f=0,001$ & $g=0,001$ & $\mathrm{~h}=0,001$ & $\mathrm{I}=0,001$ & $j=0,305$ \\
\hline \multirow[t]{3}{*}{ Ort(Gy) } & $\begin{array}{c}16,99(6,5 \\
1-29,53) \\
\end{array}$ & $\begin{array}{c}19,61(4,03- \\
28,84) \\
\end{array}$ & $\begin{array}{c}15,47(9,87- \\
19,61) \\
\end{array}$ & $\begin{array}{c}14,22(11,09 \\
-15,83) \\
\end{array}$ & $\begin{array}{c}14,05(11,35- \\
16,10) \\
\end{array}$ \\
\hline & $a=0,267$ & $\mathrm{~b}=0,775$ & $c=0,089$ & $d=0,081$ & $\mathrm{e}=0,067$ \\
\hline & $f=0,002$ & $g=0,001$ & $\mathrm{~h}=0,056$ & $\mathrm{I}=0,029$ & $j=0,367$ \\
\hline \multicolumn{6}{|c|}{ Spinal Cord } \\
\hline \multirow[t]{3}{*}{ Ort(Gy) } & $\begin{array}{c}12,02(9,4 \\
4-15,82)\end{array}$ & $\begin{array}{c}20,01(14,6- \\
27,15)\end{array}$ & $\begin{array}{c}15,25(11,80 \\
-19,88)\end{array}$ & $\begin{array}{c}18,75(12,74 \\
-25,54)\end{array}$ & $\begin{array}{c}20,81(12,45- \\
26,80)\end{array}$ \\
\hline & $a=0,001$ & $\mathrm{~b}=0,001$ & $c=0,001$ & $d=0,001$ & $\mathrm{e}=0,001$ \\
\hline & $f=0,713$ & $g=0,838$ & $\mathrm{~h}=0,002$ & $\mathrm{I}=0,001$ & $j=0,267$ \\
\hline \multicolumn{6}{|c|}{$\begin{array}{l}\text { a:3 Alan 3BKRT-4 Alan 3BKRT, b:3 Alan 3BKRT-5 Alan IMRT, c:3 Alan } \\
\text { 3BKRT-1 Ark VMAT, d:3 Alan 3BKRT-2 Ark VMAT, e:4 Alan 3BKRT-5 } \\
\text { Alan IMRT, f:4 Alan 3BKRT-1 Ark VMAT, g:4 Alan 3BKRT-2 Ark VMAT, } \\
\text { h:5 Alan IMRT- } 1 \text { Ark VMAT, I:5 Alan IMRT-2 Ark VMAT, j:1 Ark VMAT-2 } \\
\text { Ark VMAT }\end{array}$} \\
\hline
\end{tabular}

Konformite indeksi (CI) formülü olarak Van’t Riet ve arkadaşları tarafından tanımlanan;

$\mathrm{CI}=\frac{T V_{R I}}{T V} \chi \frac{T V_{R I}}{V_{R I}} 19$

formülü kullanılmıştır. Burada $\mathrm{TV}_{\mathrm{RI}}$ reçete edilen dozu alan hedef hacim, TV tedavi edilmesi gereken hedef hacim, $\mathrm{V}_{\mathrm{RI}}$ verilen dozu alan hedef hacimdir.
CI değeri 1'e eşit olduğunda ideal tümör tedavi uyumu sağlanmış olur. Pratikte " 1 " değerinin elde edilmesi mümkün olmayabilir. Değerin "1"den büyük olması durumu sadece belirlenen hedef hacmin değil çevredeki sağlıklı dokuların da 1şınlamaya maruz kaldığını, "1"den küçük olması ise hedef hacmin sadece bir kısmının ışınlanmış olduğunu ve uygun planın yapılamamış olduğunu göstermektedir ${ }^{17}$.

\section{Tartışma ve Sonuç}

Pankreas kanserleri çevresinde birçok riskli organ bulunduran ve bu nedenle tedavi planı hazırlanması oldukça zor olan kanserlerdir. Pankreas kanseri radyoterapisinde en büyük zorluk, yakın ve uzak metastaz riski nedeniyle bölgesel lenf düğümlerinin de ışınlanmasının gerekliliğinden kaynaklanmaktadır. Pankreas kanseri radyoterapisinde standart tedavi tekniği olarak kullanılan 3BKRT yönteminin yanı sıra teknolojinin gelişmesiyle birlikte tedavi yöntemlerindeki seçenekler artmıștır. IMRT, VMAT gibi tekniklerin kullanımıyla birlikte farklı tümörlerde kritik organların daha iyi korunabildiği gösterilmiştir. Yapmış olduğumuz bu çalışma da pankreas kanseri tanılı olgularda hedef hacim dozlarının ve risk altındaki kritik organların almış oldukları dozların dozimetrik karşılaştırılması değerlendirilmiş̧ir.

Chapman ve ark. 2015 yılında yaptıkları çalışmada pankreas kanserli 12 olguyu değerlendirmiş; 4 alan (AP, PA, sağ ve sol lateral) ve 5 alan (AP oblik, PA oblik, sağ ve sol lateral, inferior oblik) 3BKRT, VMAT tek ark, IMRT 5 veya 6 alan tekniklerini kullanarak planlar yapmışlar, bu teknikleri dozimetrik açıdan ve tedavi süreleri bakımından karşılaştırmayı amaçlamışlardır. Çalışmalarında bölgesel lenf nodlarına 45 Gy, tümör yatağına 50,4 Gy doz vermişler, PTV için reçete edilen bu dozun \%100‘ünün hedef hacmin \%95'ini sarmasını hedeflemişlerdir. Spinal kordun maksimum dozuna bakıldığında 3BKRT tekniğini diğer tekniklerden üstün bulmuşlardır. Kritik organlardan böbreklerin ve karaciğerin ortalama dozları açısından VMAT ve IMRT teknikleri arasında istatistiksel olarak anlamlı fark bulamamalarına rağmen, IMRT planlarında bu organların ortalama dozların daha düşük bulmuşlardır. Böbreklerin $\mathrm{V}_{15 \mathrm{G} y}, \mathrm{~V}_{18 \mathrm{G} y}$ ve $\mathrm{V}_{20 G y}$ hacimlerinin VMAT tekniğinde daha düşük olduğunu, ince bağırsak $\mathrm{V}_{35 \mathrm{~Gy}}$ ve $\mathrm{V}_{45 \mathrm{~Gy}}$ dozlarında da yine VMAT tekniğinin üstün olduğunu rapor etmişlerdir. Karaciğerin 30 Gy alan hacminde IMRT ve VMAT arasındada farklılık bulamamışlar fakat IMRT tekniğinde karaciğerin $30 \mathrm{~Gy}$ alan hacmi için dozu daha düşük bulmuşlardır. Karaciğer $\mathrm{V}_{35 \mathrm{~Gy}}$, midenin $\mathrm{V}_{35 G y}$ ve $\mathrm{V}_{45 \mathrm{~Gy}}$ değerlerinde de VMAT tekniğini daha iyi bulmuşlardır. Tedavi sürelerinin ortalama olarak 4 alan tekniğinde $3,6 \mathrm{dk}, 5$ alan tekniğinde $8,2 \mathrm{dk}$, IMRT 9,3 dk ve VMAT tekniğinde 3,1 dk olduğunu bildirmişlerdir ${ }^{20}$. Sonuç olarak Chapman ve arkadaşla- 
r1 yapmış olduğu çalışmada VMAT ve IMRT tekniklerinin kritik organlar açısından dozimetrik fayda sağladığını bildirmişlerdir. Çalışmamızda da benzer şekilde VMAT 1 ark ile VMAT 2 ark arasında karaciğerin ortalama dozu, $V_{35 G y}$ ve $V_{27 G y}$ dozları açısından istatistiksel anlamlı fark bulunamamıştır ancak VMAT 2 ark değerleri daha düşük sonuçlar vermiştir. Karaciğerin ortalama dozu için en düşük değer VMAT 2 ark tekniğinde elde edilmiştir. En yüksek değer 3 alan 3BKRT tekniğinde elde edilmiştir. 35Gy alan karaciğer hacmi için en yüksek değer 3 alan tekniğinde elde edilmiştir.

Bittner ve arkadaşları 3BKRT ve IMRT teknikleri ile radyoterapi uygulanan pankreas kanserli hastalarda gelişen radyoterapi sonrası toksisiteyi karşılaştırmayı amaçlamışlar, ama çalışmanın sonucunda önemli farklılık bulamamışlardır. Ancak tedavi ile ilgili toksisiteler, yani mide bulantısı/kusma, ishal ve geç gastrointestinal toksisite IMRT ile önemli ölçüde azalmıştır. $\mathrm{Bu}$ nedenle yapmış oldukları bu çalışmada pankreas kanseri için IMRT kullanımını önermişlerdir ${ }^{21}$.

Ali ve ark. yapmış oldukları çalışmada 10 hastaya VMAT 2 ark ve 7alan IMRT ile tedavi planları hazırlayıp bu iki tekniği dozimetrik açıdan karşılaştırmışlardır. IMRT ve VMAT teknikleri arasında PTV maksimum dozu, ortalama dozu, V95\% ve CI açısından önemli istatistiksel farklılık yoktur. Karaciğerin $\mathrm{V}_{30 \mathrm{~Gy}}$ ve $\mathrm{V}_{45 G y}$ alan hacimlerinde, ince bağırsağın $\mathrm{V}_{30 \mathrm{~Gy}}$ ve $\mathrm{V}_{45 G y}$ alan hacminde, spinal kordun $\mathrm{V}_{10 \mathrm{~Gy}}$ alan hacminde iki teknik arasında fark bulamamışlardır. Sağ ve sol böbreğin $\mathrm{V}_{15 G y}, \mathrm{~V}_{20 \mathrm{~Gy}}$ ve $\mathrm{V}_{25 \mathrm{~Gy}}$ alan hacimlerini iki teknik açısından kıyasladıklarında, VMAT tekniğinin sol böbreğin $V_{20 G y}$ ve $V_{25 G y}$, sağ böbreğin $V_{15 G y}$, $\mathrm{V}_{20 \mathrm{~Gy}}$ ve $\mathrm{V}_{25 \mathrm{~Gy}}$ alan hacimlerinde istatistiksel olarak önemli düşüş gösterdiğini yani VMAT tekniğinin bilateral böbrek dozunu iyileştirdiğini bildirmişlerdir ${ }^{22}$. Yapmış olduğumuz çalışma da böbreklerin $\mathrm{V}_{12 \mathrm{~Gy}}$ ve $\mathrm{V}_{18 G y}$ açısından VMAT 2 ark ile VMAT 1 ark planları arasında istatistiksel olarak anlamlı farklılık bulunamamıştır ancak VMAT 2 ark planlarında diğer tekniklerden daha iyi değerler elde edilmiştir. Yerleşim yeri bakımından böbrekler ve karaciğer toksisitesi açısından radyoterapi planlaması için zor olan pankreas tümörleri için 3BKRT planları yapılırken bu OAR'ların dozlarının sınırda tutulması zor olmaktadır. Çalışmamızda karaciğerin 27 Gy alan hacmi açısından IMRT 5 alan tekniği 3BKRT tekniklerinden daha düşük ancak VMAT tekniklerimizden daha yüksektir ve VMAT 2 ark tekniği karaciğer $\mathrm{V}_{27 G y}$ açısından da daha üstündür. CI, HI açısından IMRT ile 3BKRT ve VMAT 1 ark ile VMAT 2 ark teknikleri arasında farklılık yoktur, fakat en uygun HI ve CI değerlerini VMAT 2 ark tekniği vermiştir.

Geld ve ark. pankreas kanserli hastalarda 3BKRT tekniği, IMRT tekniği ve solunuma duyarlı radyoterapi tekniklerini karşılaştırmışlardır. IMRT tekniğinin aynı zamanda mide ve ince bağırsak dozlarında da azalma sağladığı görülerek IMRT tekniğinin sağ böbrek, karaciğer, mide ve ince bağırsak dozlarında gözle görülür bir azalma sağladığını rapor etmişlerdir. Elde edilen sonuçlarda IMRT tekniğinin 3BKRT tekniğine kıyasla ortalama sağ böbrek dozunda kayda değer azalma sağladığını ancak aynı başarının sol böbrek için geçerli olmadığını vurgulamışlardır ${ }^{23}$. Çalışmamızda tümör yerleşimi sağ tarafta olan hasta planlarında hem 3BKRT tekniği hem de IMRT tekniği böbrek dozunu koruma açısından başarılı olamamışken, VMAT teknikleri bunu sağlayabilmiş ve VMAT 2 ark tekniğinde doz daha düşük bulunmuştur. Sol böbrek için tüm teknikler doz sınırlamalarını sağlamış, 3BKRT tekniği daha iyi sonuç vermiştir. IMRT tekniğinde tüm böbrek dozları daha yüksek ve VMAT teknikleri arasında fark olmamasına rağmen VMAT 2 ark tekniğinde doz diğer tekniklere göre daha düşük bulunmuştur. Bunun nedeni farklı planlama programlarının kullandığı farklı algoritmalardır. VMAT tekniğinde Monte Carlo algoritması kullanılmakta böylece kritik organlar daha iyi korunmaktadır. Her iki böbreği beraber değerlendirdiğimizde VMAT planlarının klinik olarak daha iyi olduğu uygun görülmüştür.

Benzer şekilde, Nabavizadeh ve arkadaşları 2014 yılında değerlendirdikleri 20 pankreas hastasına 3BKRT'de 4 ve 6 alan, IMRT'de 6 ve 9 alan ve VMAT'da 2 ark kullanarak planlar oluşturmuşlardır. Tüm planlarını 10 Mv x-ışını kullanarak yapmışlar, ilk planda 25 fraksiyonda 45 Gy, ikinci plana ise 8 fraksiyon boost dozu vererek 59,4 Gy'e çıkmışlardır. Yaptıkları çalışmada sonuç olarak, pankreas kanseri radyoterapisinde 3BKRT ve IMRT ile kiyaslandığında VMAT'ın dozimetrik yarar sağlamakta olduğunu ve 59,4 Gy'in bu hastalarda kritik organlarda doz kısitlamasına rağmen uygulanabilir olduğunu bildirmişler$\operatorname{dir}^{5}$.

Glowacki ve arkadaşları yaptıkları çalışmada 3BKRT tekniğinde 2 alan, 3 alan, 4 alan ve IMRT tekniklerini karşılaștırmışlardır. 3BKRT'ye kıyasla IMRT'de böbreklerin daha iyi korunduğunu, bağırsak ve karaciğer korunması açısından iki tekniğinde kabul edilebilir ve benzer olduğunu bildirmişlerdir ${ }^{24}$. Yerleşim yeri bakımından böbrekler ve karaciğer toksisitesi açısından radyoterapi planlamasının zor olduğu pankreas tümörleri için 3BKRT planları yapılırken bu OAR'ların dozlarının sınırda tutulması güç olmaktadır. Çalışmamızda karaciğerin 27 Gy alan hacmi açısından IMRT 5 alan tekniği 3BKRT tekniğinden daha düşük ancak VMAT tekniklerimizden daha yüksektir ve VMAT 2 ark tekniği karaciğer $\mathrm{V}_{27 \mathrm{~Gy}}$ açısından daha üstün sonuçlar vermiştir.

Brown ve arkadaşları 15 hastayı incelerek yaptıkları çalışmada, 3BKRT, IMRTs (SIB ile 27fx) ve IMRTi (Boostlu) tekniklerini dozimetrik açıdan karşılaştırmışlardır. IMRT tekniği ile 20 Gy alan toplam böbrek hacminde, 35 Gy alan karaciğer hacminde ve 45 Gy alan ince bağırsak hacminde 3BKRT tekniğine göre 


\section{B. İbicioğlu, ark.}

daha düşük değerler bulduklarını vurgularken spinal kord dozlarında bir farklılık olmadığını bildirmişlerdir. Sonuç olarak IMRT tekniğinin 3BKRT tekniğine göre tümör hacmine gerekli dozu verirken sağlıklı dokuları daha iyi koruduğunu rapor etmişlerdir ${ }^{25}$. Çalışmamızda planlar yapilırken, spinal kordun maksimum dozunun 45 Gy'i geçmemesi hedeflenmiş ve tüm planlarda bu değer sağlanabilmiştir. Spinal kordun ortalama dozu değerlendirilmiş, 3 alan 3BKRT dişında teknikler arasında çok fark bulunamamıştır. Bu teknikde arkadan gelen ışın olmadığından 3BKRT 3 alan tekniğinin daha iyi sonuç verdiği saptanmıştır.

Sonuç olarak, bu çalıșmada pankreas tümörü tedavisinde 3 farklı teknik ile 5 farklı tedavi planlaması kullanılarak kritik organ dozları ve hedef hacimler karşılaştırılmıştır. Pankreas tümörleri yerleşim yeri olarak böbrekler ve karaciğer gibi kritik organlara yakın olması nedeniyle 3BKRT planları karaciğer açısından kabul edilebilir; ancak böbrekleri koruma açısından yeterli değildir. VMAT 2 ark tekniği diğer tekniklere göre dozun hedefi sarımı, CI, HI ve kritik organ dozları açısından üstünlük göstermektedir. $\mathrm{Bu}$ sonuçlarla oluşabilecek yan etkiler göz önüne alınd1ğında tedavi tekniği olarak VMAT tercih edilebilir bir tedavi tekniğidir ve "set-up" süresinin kısalığı uygulamayı hızlandırmaktadır.

\section{Kaynaklar}

1. Murat Tuncer ve ark. Türkiye'de Kanser Kontrolü. T.C. Sağlık Bakanlığı. 45-49. (2009).

2. Siegel R, Ma J, Zou Z, Jemal A. Cancer statistics, CACancer J Clin 2014;64(January-February (1)):9-29.2014

3. Ma J, Siegel R, Jemal A. Pancreatic Cancer Death Rates By Raceamong US Men and Women;1970-2009. Journal of the National Cancer Institute, 2013;105(22):1694-1700.

4. Kelsen DP. Principles and Practice of Gastrointestinal Oncology. 2nd edition. Philadelphia: Lippincott Williams \& Wilkins Pres; 2008. 294-295.

5. Nabavizadeh N, Simeonov AO, Waller JG, Romer JL, et al. Volumetric-Modulated Arc Radiotherapy For Pancreatic Malignancies: Dosimetric Comparison With Sliding-Window Intensity-Modulated Radiotherapy and 3-Dimensional Conformal Radiotherapy. Medical Dosimetry, 2014;39(3):256-260.

6. Loehrer SR PJ, Feng Y, Cardenes H, et al. Gemcitabine Alone Versus Gemcitabine Plus Radiotherapy In Patients With Locally Advanced Pancreatic Cancer: An Eastern Cooperative Oncology Group Trial. Journal of Clinical Oncology, 2011;29(31):4105-4112.

7. Hammel P, Huguet F, Van Laethem J, et al: Comparasion of Chemoradiotherapy (CRT) and Chemotherapy (CT) In Patients With A Locally Advanced Pancreatic Cancer (LAPC) Controlled After 4 Months of Gemcitabine With or Without Erlotinib: Final Results of The International Phase III LAP 07 Study. Journal of Clinical Oncology, 2013;(31):2156-2165.

8. Henry AM, Ryder WDJ, Moore C, et al. Chemoradiotherapy For Locally Advanced Pancreatic Cancer: A Radiotherapy Dose Escalation and Organ Motion Study. Clinical Oncology, 2008;20:541-547.
9. Philips TLP. Leibel's Textbook of Radiation Oncology. 3th edition, Philadelphia. Elsevier Saunders Press, 2010;156-172.

10. Abeloff MD. Abeloff's Clinical Oncology. 4th edition, Philadelphia. Churchill Livingstone Elsevier Press, 2008;1117-1135.

11. 11.Willett, C.G.; Czito B.G.; Bendell, J.C. Cancer of the pancreas. In: Halperin, E.C.; Perez, C.A.; Brady, L.W., editors. Principles and Practice of Radiation Oncology. 6th Edition, Philadelphia.Lippincott Williams\& Wilkins Press, 2008;

12. Regine WF, Winter KA, Abrams RA, et al. Fluorouracil vs Gemcitabine Chemotherapy Before and After FluorouracilBased Chemoradiation Following Resection of Pancreatic Adenocarcinoma: A Randomized Controlled Trial. Journal of American Medical Association, 2008;299:1019-26.

13. Agulnik M. Puataweepong P, (eds). Advanced Radiation Therapy for Head and Neck Cancer: A New Standard of Practise. 1st Edition. Thailand Intech Press, 2012;232-235.

14. Wang X, Zhang Y, Bai S, et al. Single-Arc VolumetricModulated Arc Therapy (Svmat) As Adjuvant Treatment For Gastric Cancer: Dosimetric Comparasions With ThreeDimensional Conformal Radiotherapy (3D-CRT) And İntensity-Modulated Radiotherapy. Medical Dosimetry, 2013;38(4):395-400.

15. Otto K. Volumetric modulated arc therapy: IMRT in a single gantry arc. Medical Physics, 2008;35(1): 310-317.

16. Wambersie A, Deluca PM, Caswell RS and Menzel HG. The International Commission On Radiation Units And Measurements (ICRU) Activities and Future Plans. ICRP Publication 60. Maryland, Pergamon Press, 2007;20-22.

17. Feuvret L, Noël G, Mazeron JJ and Bey P. Conformity index: a review. International Journal of Radiation Oncology * Biology* Physics, 2006;64(2):333-342.

18. ICRU (International Commission on Radiation Units and Measurements), Prescribing, Recording and Raporting Electron Beam Therapy, Report No.71, Bethesda, MD.2009

19. Van't Riet A, Mak AC, Moerland MA, Elders LH and Zee W, et al. A Conformation Number to Quantify The Degree of Conformality In Brachy Therapy and External Beam Irradiation: Application to The Prostate. International Journal of Radiation Oncology Biology Physics 1997;37(3):731-736.

20. Chapman KL, Witek ME, Chen H, et al. Pancreatic Cancer Planing: Complex conformal vs Modulated Therapies. Medical Dosimetry, 2016;41(2):100-104.

21. Bittner MI, Grosu AL and Brunner TB. Comparison of Toxicity After IMRT and 3D- Conformal Radiotherapy For Patients With Pancreatic Cancer- A Systematic Review. Radiotherapy and Oncology, 2015;114(1):117-121.

22. Arif NA, Aness HD, Christie SJ, Arsalan KS, and Jerome CL. Dosimetric Comparison of Volumetric Modulated Arc Therapy and Intensity-Modulated Radiation Threapy For Pancreatic Malignancies, Medical Dosimetry, 2011;37:271-275.

23. Van Der Geld YG, Van Triest B, Verbakel WF et al. Evaluation of Four-Dimensional Cmoputed Tomograpy- Based IntensityModulated and Respiratory-Gated Radiotherapy Techniques for Pancreatic Carcinoma, International Journal of Radiation Oncology, Biology, Physics, 2008;72:1215-1220.

24. Glowacki G, Wesolowska I, Wolny E, et al. The Comparison of The Conformal Radiotherapy (CFRT-2,3 and 4 fields) and Intensity Modulated Radiotherapy (IMRT) in Adjuvant Radiochemotherapy for Patients with Pancreas Cancer. EJC Supplements, 2009;2(7):386.

25. Brown MW, Ning H, Arora B, et al. A Dosimetric Analysis of Dose Escalation Using Two Intensity-Modulated Radiation Therapy Techniques in Locally Advanced Pancreatic Carcinoma. International Journal of Radiation Oncology* Biology* Physics, 2006;65(1):274-283. 